(U) $O$ Observatori de

Bioètica i Dret

ISSN: 1886-5887

\section{Revista de Bioética y Derecho}

Publicación del Máster en Bioética y Derecho nww.bioeticayderecho.ub.es

BiOÉTICA ANIMAL

\title{
Comentarios sobre la Directiva Europea 2010/63/EU para la protección de animales de laboratorio
}

\author{
JORDI L. TREMOLEDA*
}

* Dr. Jordi L. Tremoleda DVM, MSc, PhD, MRCVS. Licenciado en Medicina Veterinaria (UAB, Barcelona, ES), Máster en Bioética (PCB, UB, Barcelona, ES), PhD in Veterinary Sciences (Utrecht University, NL), Miembro del Royal College of Veterinary Surgeons, London, UK (MRCVS). jordi.lopez-tremoleda@imperial.ac.uk 


\section{Resumen}

El 20 de Octubre del 2010 se publico la nueva Directiva Europea 2010/63/EU sobre la protección de los animales utilizados en procedimientos científicos. Dicha publicación en el Diario Oficial de la Unión Europea supone el inicio de su tramitación para su entrada en vigor como ley Europea. Los países miembros tienen ahora un periodo de dos años para implementar dicha directiva como documento legal asegurando su completa entrada en vigor para Enero del 2013. En este artículo se realiza un análisis comparativo de la nueva Directiva 2010/63/EU y la normativa actual vigente en el Reino Unido, referente mundial en el área de protección de animales de laboratorio. La nueva Directiva reconoce la importancia de la utilización de animales de experimentación en los avances científicos, pero reforzando la defensa y el respeto del valor intrínseco del ser animal. En este sentido la Directiva representa un importante avance para la protección del bienestar animal en todos los estados miembros, con el objetivo de armonizar la legislación vigente. Si bien varios países ya disponen de una legislación bastante progresiva, la implementación de la nueva directiva representa una excelente oportunidad para asentar y/o homogeneizar criterios de alto estándar de protección del bienestar animal en todos los países miembros, reforzando así el compromiso de la Unión Europea con la investigación científica y el respeto al bienestar de los animales de experimentación.

Palabras clave: experimentación animal; directiva; EU; bienestar animal.

\section{Abstract}

On 20th October 2010 the new 2010/63/EU European directive on the protection of animals used in scientific procedures was published in the Official Journal of the European Union, entering into force as a European law. Member States now have a period of two years to implement the directive as a legal document ensuring its full legal binding by January 2013. This article undertakes a comparative analysis between the new directive 2010/63/EU and the current regulatory system in the UK, which is an international reference in the protection of laboratory animals. The new directive recognizes the importance of the use of animal experiments in scientific advances, but ensuring the defence and respect for the intrinsic values of the animals. In this regard the directive represents an important advance for the protection of animal welfare in all the Member States, with the aim of harmonizing such values in the EU current legislation. While several countries already have fairly progressive legislation, the implementation of the new directive represents an excellent opportunity to reinforce high criteria levels in the defence of animal welfare throughout all the Member States. This represents a good opportunity for the EU to reinforce its commitment with the scientific research and the welfare of experimental animals.

Key words: animal experimentation; directive; EU; animal welfare. 


\section{Introducción a la legislación vigente}

En 1986 se adopto la Directiva Europea EU 86/609¹ para la protección de los animales utilizados para experimentación, con el objetivo de mejorar el control de los animales de experimentación y establecer estándares mínimos sobre su alojamiento y la preparación del personal que trabaja con animales de laboratorio. Con los avances científicos, la sociedad ha ido desarrollado una actitud mucho más crítica con respecto a varios aspectos ético-sociales relacionados con la investigación y su aplicación para el desarrollo de nuevas terapias. En particular, esto se refleja en la adopción de posiciones mucho más progresivas en defensa del bienestar animal. Este interés social se ve proyectado con la implementación de varios tratados europeos; el Tratado de Amsterdam de 1997 ya incluye un protocolo para el bienestar animal ${ }^{2}$ y en 1998 se aprueba la entrada en vigor de la Decisión 1999/575/EC de la Convención Europea para la protección de los animales vertebrados utilizados en experimentación y otros procedimientos científicos ${ }^{3}$. Paralelamente también se establece el Centro Europeo para la validación de métodos alternativos ${ }^{4}$ (ECVAM), que refuerza el interés de la Comisión Europea para la protección del bienestar animal, y en concreto, promueve nuevas discusiones sobre el uso de animales de experimentación en investigación e industria biotecnológica y medica. Todo esto conlleva en el 2002 la decisión del Parlamento Europeo de revisar la Directiva EU/86/609. En 2008 se establece las recomendaciones para la revisión de la Directiva, con una importante participación no solo de grupos de expertos profesionales del área académica, industria e instituciones gubernamentales, sino también la presencia de grupos profesionales de defensa de la salud y bienestar animal, como el Comité Científico en sanidad Animal y Bienestar Animal (SCAHAW) ${ }^{5}$.

La nueva Directiva Europea 2010/63/EU ${ }^{6}$ fue presentada en Noviembre 2010 a los diferentes miembros de la UE, culminando así casi 10 años de trabajo por parte de la Comisión Europea. Se inició así el periodo de "transposición" durante el cual los países miembros tendrán que implementar esta Directiva en su legislación nacional. Durante este periodo de 2 años los países miembros han de implementar todas las medidas administrativas, logísticas e informativas que permitan la aprobación de la nueva legislación. Esto implica que los países tendrán que adaptar y reformar su legislación

\footnotetext{
1 Council Directive 86/609/EEC of 24 November 1986 on the approximation of laws, regulations and administrative provisions of the Member States regarding the protection of animals used for experimental and other scientific purposes http://eur-lex.europa.eu/LexUriServ/LexUriServ.do?uri=CELEX: 31986L0609: en: HTML

2 Treaty of Amsterdam amending the treaty on European Union, the treaties establishing the European Communities and related acts. Protocol on protection and welfare of animals Official Journal C 340, 10 November 1997 http://eur-lex.europa.eu/en/treaties/dat/11997D/htm/11997D.html

3 1999/575/EC: Council Decision of 23 March 1998 concerning the conclusion by the Community of the European Convention for the protection of vertebrate animals used for experimental and other scientific purposes http://eur-lex.europa.eu/LexUriServ/LexUriServ.do?uri=CELEX:31999D0575: EN: NOT

${ }^{4}$ European Centre for the Validation of Alternative Methods http://ecvam-dbalm.jrc.ec.europa.eu/

${ }^{5}$ Scientific Committee on Animal Health and Welfare http://ec.europa.eu/food/fs/sc/scah/index_en.html

${ }^{6}$ European Directive 2010/63/EU

http://eur-lex.europa.eu/LexUriServ/LexUriServ.do?uri=OJ:L: 2010:276:0033:0079: En:PDF
} 
vigente referente a la protección de animales utilizados en procedimientos científicos. La nueva directiva representa una buena oportunidad para armonizar la regulación de la utilización de animales en investigación entre los países miembros, asegurando una buena estandarización de protocolos y de valoraciones éticas y de protección del bienestar animal. Indudablemente esto supone un importante compromiso para los países miembros, especialmente para aquellos países con unos niveles de regulación menos restrictivos que tendrán que demostrar su compromiso para implementar los principios de la nueva directiva.

Los principales objetivos que han llevaron al desarrollo de la nueva Directiva son:

- El armonizar la legislación vigente referente al uso de animales de laboratorio entre los países miembros de la UE, facilitando así una estandarización entre toda la comunidad científica y los laboratorios de los diferentes estados.

- El reforzar la protección de los animales utilizados en investigación.

En base a estos principios de armonización y estandarización, y el reforzamiento del bienestar animal propuestos, la impresión general de la nueva Directiva es positiva, tal y como lo refleja la valoración presentada por la comisión de trabajo del Comité de Ciencias Medicas de la European Science Foundation y de la European Medical Research Councils ${ }^{7}$. Esta nueva legislación continúa promoviendo el responsable uso de animales en investigación para el beneficio de los avances medico-científicos, preservando las mejores condiciones para el bienestar animal. Asimismo se pide la máxima participación de la comunidad científica para asegurar la implementación de la nueva Directiva en las legislaciones de los diferentes países miembros.

Es una buena oportunidad para centralizar la regulación del uso de animales para investigación entre todos los países miembros de un modo mucho más eficiente, facilitando la interacción entre centros de investigación y la industria farmacéutica en la UE.

Grupos científicos y de profesionales de los países miembros trabajando en el área de bienestar animal y su uso para investigación están llevando a cabo varias consultas sobre la transposición de la nueva Directiva y como su implementación va a afectar la legislación vigente. En particular en el Reino Unido, donde ya existe un legislación exhaustiva para la protección de animales utilizados en procedimientos científicos $[A(S P) A]^{8},{ }^{9}$ y un sistema gubernamental de inspectorado bien asentado, el gobierno ha llevado a cabo varias consultas $^{10},{ }^{11}$ para asegurar que la

\footnotetext{
${ }^{7}$ Position on the Directive on the Protection of Animals used for scientific Purposes (2010/63/EU) http://www.codex.uu.se/texts/ESF-EMRC\% 20position\%20paper\%20on\%20Directive\% 20201063EU.pdf

${ }^{8}$ Animal (Scientific Procedures) Act 1986

http://tna.europarchive.org/20100413151426/http://www.archive.officialdocuments.co.uk/document/hoc/321/321-xa.htm

${ }^{9}$ Guidance on the Operation of the Animals (Scientific Procedures) act 1986 http://tna. europarchive.org/20100413151426/http://www. archive. officialdocuments.co.uk/document/hoc/321/321.htm

10 Transposition of European directive 2010/63/EU - protection of animals used for scientific purposes http://www. homeoffice.gov.uk/publications/about-us/consultations/transposition-protection-animals/
} 
implementación de la nueva Directiva no compromete la eficacia del sistema actual en defensa del bienestar animal.

La intención de este documento es hacer un resumen de los elementos principales de la nueva Directiva e informar de la áreas que son consideradas más críticas, comparándola con la legislación vigente en el Reino Unido, una de las más desarrolladas en el área de protección de los animales de experimentación.

Nueva Directiva 2010/63/EU: Elementos claves

- Más extensa y con más detalle que la Directiva existente de 1986

- Define claramente los animales y su estado de desarrollo permitidos, con especial valoración para animales salvajes, primates no-humanos, perros y gatos

- Describe con detalle los objetivos permitidos en base a la aplicación de las 3Rs (reemplazo, reducción y refinamiento) de Russell \& Burch ${ }^{12}$, selección de métodos, valoración del grado de severidad, métodos de eutanasia, etc...

- Define los establecimientos para la cría y desarrollo de animales de experimentación, citando todos los requerimientos de personal, equipo, alojamiento, monitorización y grupos de protección del bienestar animal ("animal welfare bodies").

- Describe los requisitos de preparación, educación y competencias del personal involucrado, estableciendo un sistema de supervisión mediante un cuerpo de autorización estatal.

- Establece que los proyectos de investigación con animales tienen que estar evaluados y aprobados por la autoridad competente, mediante el sistema de autorización estatal.

\section{Áreas críticas}

\section{Establecimiento de autoridades competentes y medidas más restrictivas}

Cada país deberá asignar una autoridad que supervise de una manera objetiva, crítica y exigente la implementación de la Directiva. El Reino Unido ya dispone de un sistema legislativo bien establecido [Animals (Scientific Procedures Act 1986; A (SP) A] ${ }^{\text {viii, iv }}$, a través de un cuerpo nacional de inspectores cuya función es la de supervisar la implementación de la ley vigente. Esta es una los puntos que han suscitado un importante debate sobre que instituciones son las encargadas de implementar la nueva Directiva y asegurar un seguimiento retrospectivo de la normativa. La Directiva

\footnotetext{
${ }^{11}$ Summary of responses to the Consultation on proposals for the revision of EU Directive 86/609/EEC on the proteccion of animals used for scientific purposes.

http://webarchive. nationalarchives.gov.uk/20100413151426/http://scienceandresearch.homeoffice.gov.uk/ani mal-research/legislation/summary-report2835.pdf?view=Binary

12 The 3Rs principles were first introduced in Russell and Burch's 1959. Book 'The principles of humane experimental technique'. which is available in full text on the Altweb site at: 
permite a estados miembros la posibilidad de mantener sus sistemas vigentes, en particular en aquellos casos que ya exista una legislación extensiva que preserve el bienestar animal, evitándose así que dichos países se vean forzados a diluir sus sistemas regulatorios ya existentes. En este sentido la Directiva establecería una homogeneización de medidas mínimas en los países miembros, pero dando permisividad para que cada país establezca medidas más estrictas.

En referencia al grado de severidad de los procedimientos aceptados, cada proceso deberá ser valorado individualmente, en base al grado de dolor, sufrimiento, stress o efectos a largo plazo que se puedan causar en cada animal durante el proceso de experimentación. Según estos criterios, la valoración de severidad será establecida por cada caso en base a juicios profesionales y científicos. La normativa evita así establecer una lista específica de procedimientos. Pero por otra parte de este modo persiste el riesgo de que la valoración pueda ser demasiado subjetiva, basándose en la opinión de ciertos individuos.

La Directiva establece algunos ejemplos sobre la clasificación de severidad de procesos, pero varios grupos en el Reino Unido (LASA) ${ }^{13}$ se muestran críticos porque los casos expuestos no reflejan todas situaciones, en particular en referencia a procedimientos de severidad alta. Se cita que "cualquier procedimiento que conlleve dolor, sufrimiento o stress severo y de largo efecto y que no pueda ser minimizado deberá ser evitado"; esta citación da pie a interpretaciones confusas sobre todo en lo referente a la utilización de modelos animales para la investigación del dolor. Es importante clarificar la protección de los estudios con este tipo de modelos animales, donde el dolor crónico es parte de su sintomatología (por ejemplo, modelos de dolor neuropatico con lesiones nerviosas o de dolor asociados al desarrollo de cáncer. LASA recomienda que se incluyan información más detallada sobre clasificación de severidad en base a recomendaciones de un Comité de Expertos, evitando así la ambigüedad de interpretación entre países miembros.

\section{Definición del uso de los animales y procedimientos}

En referencia a cómo y que animales deberían ser utilizados en los procedimientos experimentales, el uso de primates no-humanos se mantiene restringido a aquellos procedimientos aplicados para la prevención, diagnóstico o tratamiento de enfermedades debilitantes o con un alto riesgo para la salud humana (HIVIAIDS, malaria, enfermedades neurodegenerativas). En este sentido se intenta evitar la utilización de primates en aquellos estudios donde la utilización de otros modelos animales como ratones se ha demostrado efectiva, como son varias áreas de investigación de ciencia básica y estudios farmacológicos, fisiológicos y/o genéticos. Pero en la nueva directiva no queda bien definido quien ni de qué manera se va a valorar qué tipo de procedimientos son necesarios. Por ejemplo, la definición de enfermedad debilitante conlleva gran ambigüedad, abarcando un gran escenario de condiciones patológicas.

\footnotetext{
13 Laboratory Animals Science Association (LASA) : response to the Home Office consultation to the transposicion of the Directive 2010/63/EU on the protection of animals use for scientific purposes. http://www. lasa.co.uk/LASA\% 20consultation\%20response\%202010-63-EU.pdf
} 
La directiva cubre el procedimiento para la eutanasia de los animales de laboratorio, especificando el uso de técnicas aprobadas y que induzcan el mínimo dolor, sufrimiento y stress en los animales. También describe la posibilidad de utilizar otros medios alternativos, siempre que haya una base científica que demuestre que estos métodos alternativos sean considerados también de modo humanitario. Desafortunadamente no se describen ejemplos de estos métodos alternativos, lo que también puede conllevar cierto grado de ambigüedad. Por ejemplo, no se incluyen métodos que son utilizados con cierta frecuencia para eutanasia de animales de laboratorio, como es el caso de la inducción de hipotermia para el sacrificio de roedores o aves en estadio neonato, la dislocación cervical sin sedación cervical en casos de emergencia y/o determinadas situaciones experimentales que no permitan el uso de sedantes por su efecto neurotrópico, o la irradiación por microondas que es una técnica muy utilizada en neurobiología experimental ${ }^{14}$. Estas metodologías aplicadas de modo adecuado son consideradas como procedimientos adecuados en varias legislaciones nacionales de países miembros; la nueva directiva no prohíbe su utilización pero tampoco describe de forma clara que autoridad tendrá que regular su aprobación y monitorización en aquellos casos que sea solicitado.

La re-utilización de animales está restringida a procesos de severidad moderada, por ejemplo, extracción de sangre, canulación de vasos superficiales o extracción de pequeñas biopsias superficiales. Estos procesos no conllevan gran dolor, sufrimiento y/o estrés prolongado y su confirmación deberá estar apoyada por aprobación del veterinario/a. La directiva no cita de forma explícita en qué casos se permitirá la reutilización de animales, pero establece que dicha responsabilidad dependerá de la autoridad competente de los países miembros. Este punto es bastante delicado porque la re-utilización de animales de experimentación es un aspecto muy ligado a la sensibilidad pública.

\section{Cría y mantenimiento de los animales de laboratorio}

La nueva directiva confirma que los establecimientos que crían y desarrollan animales de experimentación han de estar autorizados y registrados con la autoridad competente. Varias autoridades estatales ya disponen de un registro de establecimientos certificados. La nueva directiva es menos prescriptiva en relación al procedimiento para solicitar autorización para la cría y mantenimiento de animales de laboratorio; en general se simplifica la burocracia administrativa y se da un enfoque más importante a la regulación del bienestar animal.

Los requisitos para las dimensiones de las jaulas permitidas, son expuestos de modo mandatorio en el Anexo III de la Directiva y supone una modificación con respeto a las recomendaciones establecidas en 2005 por una comisión de expertos ${ }^{15}$. Este factor puede tener un gran impacto económico en el coste para criar y mantener los animales de experimentación.

\footnotetext{
${ }^{14}$ Articulo 6 y Anexo IV de la Directivo 2010/63/EU

http://eur-lex.europa.eu/LexUriServ/LexUriServ.do?uri=0] : L: 2010:276:0033:0079: En: PDF

${ }^{15}$ European Convention for the Protection of Vertebrate Animals used for Experimental and Other Scientific
} 


\section{Personal: responsabilidades y formación}

En la Directiva no se exige de modo explícito la autorización a personal cualificado ni de supervisión de los procedimientos; pero sí que establece la necesidad de personal competente. Se presupone así que es responsabilidad del país miembro asegurar que el personal está capacitado para dicho trabajo. De este modo se intenta minimizar un sistema excesivamente administrativo y burocrático y se da más flexibilidad a los países miembros para establecer sus sistemas de preparación de personal. En el Reino Unido ya existe un sistema bien organizado de formación de personal, basado en la disposición de licencia personales ${ }^{16}$ a personal que ha ejecutado los cursos preparatorios. Tal y como se establece en el Animals (Scientific Procedures) Act 1986 [A (SP) A] Viii, el uso de animales de laboratorio requiere la obtención de tres licencias: una licencia personal para todo aquella personal que realice cualquier proceso regulado por el $[A(S P) A]$, una licencia que autoriza un programa de investigación y un certificado de designación para el lugar donde se va a realizar el trabajo con animales de laboratorio. La obtención de la licencia personal requiere la asistencia obligatoria a un curso de acreditación y su validación por parte de la Home Office (entidad gubernamental). En el sistema británico se da una gran importancia a la preparación para obtener dichas licencias como eje principal para asegurar los altos estándares del trabajo con animales de experimentación. Asimismo se establece que tanto los individuos como los centros de investigación con animales de laboratorio tienes una importante responsabilidad en asegurar que el personal es competente y ha realizado todos los cursos de formación requeridos y las instituciones han de asegurar todo personal dispone de licencia personal, que su actividad está integrada en una licencia de proyecto aprobada y que se cumplen todas las normativas referentes al mantenimiento de los animales. Este sistema permite al inspectorado de la Home Office mantener un buen control sobre los centros e individuos trabajando con animales de laboratorio, pero a la vez es criticado por ser excesivamente burocrático y poco flexible. The Laboratory Animal Science Association (LASA) XII valora positivamente la necesidad de asegurar una buena armonización de los programas de formación entre investigadores e instituciones de la UE para asegurar la movilidad de investigadores y/o proyectos bajo los mismos estándares. Pero también especifica que dichos programas de formación han de ser aprobados por mutuo acuerdo entre los países miembros y defienden el mantenimiento de un sistema regulatorio similar al ya existente en el Reino Unido. La mejor opción sería que las bases de la formación fueran establecidas a través de la Federación Europea de Asociaciones Científicas de Animales de Laboratorio (FELASA) ${ }^{17}$. Asimismo LASA también requiere el establecimiento de un sistema de evaluación retrospectiva, para asegurar una los proyectos y el personal trabajando en ellos sigue manteniendo los niveles de calidad necesarios. En esta línea si bien la Directiva reitera la responsabilidad de los individuos trabajando con animales de laboratorio,

Purposes Council of Europe ETS 123, 2005. http://conventions.coe.int/Treaty/en/Treaties/html/123.htm

16 Home Office, UK. Personal Licences application http://www.homeoffice.gov.uk/science-research/animalresearch/personal-apps/

17 Guidelines for continuing education for persons involved in animal experiments (2010) http://www.felasa.eu/recommendations/guidelines/guidelines-for-continuing-education-for-persons-involvedin-animal-experime/ 
es importante reafirmar la necesidad para mantener un sistema de valoración continuado, retrospectivo y dinámico.

Otro aspecto interesante es que la Directiva, a diferencia de lo establecido en el [A (SP) A], permite el uso de animales para proyectos de formación dando así un mejor grado de flexibilidad en el procesos de formación.

Finalmente, a diferencia del sistema ingles que regula de modo más estricto la presencia de personal requerido en el certificado de designación de establecimiento, la Directiva no describe explícitamente las responsabilidades específicas del personal. Se cita de modo más general la necesidad de tener personal capacitado para varias responsabilidades. En el Reino Unido la actual legislación exige la presencia de un responsable para supervisar el bienestar y cuidado de los animales (Named Animal Care and Welfare Officer) y la presencia de personal veterinario (Named Veterinary Surgeon) para cada establecimiento de cría, mantenimiento y manejo de animales de experimentación. De este modo, con la nueva legislación, dependerá de las instituciones asegurarse que el personal está cualificado, y ejecuta su trabajo en base a la reglamentación establecida. Será interesante ver como otros países establecen sus sistemas de formación de personal. En cierto grado, se pierde así la posibilidad de establecer un programa de formación consistente entre los países de la UE.

\section{Comité de supervisión del bienestar animal}

La directiva establece la creación de un grupo de supervisión del bienestar animal "Animal Welfare Body"), incluyendo al personal responsable del cuidado y del bienestar de los animales y al investigador responsable del proyecto. La Directiva cita que este comité podrá recibir consejos del veterinario responsable pero no se exige la presencia del profesional. Las responsabilidad del comité se centran en aconsejar al personal que trabaja con animales de laboratorio sobre aspectos relacionados con el bienestar animal, promover la aplicación de los principios de reemplazo, reducción y refinamiento en los procedimientos con animales de laboratorio ("3Rs"xii), establecer un sistema de control interno para monitorizar y valorar de modo continuo el bienestar de loa animales utilizados, hacer un seguimiento de los proyectos de investigación valorando el uso y el bienestar de los animales y promover la mejoría de las condiciones de alojamiento de los animales. La creación de este comité es uno de los ejes principales de esta Directiva y supone un importante paso para preservar y defender el bienestar animal y armonizar su regulación entre los países miembros. No obstante esta en una de las áreas más discutidas en la consulta que ha realizado el gobierno británico sobre la nueva directiva ${ }^{\mathrm{XI}}$, ya que se presupone que este grupo reemplaza al actual comité ético de experimentación animal (Ethical Review Process ; ERP ${ }^{18}$ ). Este comité ético es considerado como uno de los principales ejes de la legislación sobre animales de experimentación en el Reino

\footnotetext{
${ }^{18}$ The Ethical Review Process. Home Office, UK 1998 
Unido, con un rol importante en preservar el bienestar de los animales y asegurar que los proyectos de investigación promueven las "3Rs" y promoción de sistemas alternativos a la investigación animal.

La nueva Directiva describe que el nuevo grupo de bienestar animal tiene que incluir al menos una persona responsable del bienestar y cuidado de los animales, y una persona con conocimientos científicos. Esta posición reduce a mínimos el personal del actual comité ético, que requiere estar formado por el veterinario responsable de los animales de experimentación, personal técnico de mantenimiento de los animales, personal científico, personal no relacionado con la institución científica e inspectores gubernamentales. Estos requisitos para el Comité de bienestar animal con una importante reducción de la participación de personal altamente cualificado han sido bastante discutidos en la consulta realizada por el gobierno británico ${ }^{{ }^{i}}$, principalmente motivado por las reacciones de asociaciones en defensa de bienestar animal como LASA' ${ }^{\text {xii }}$ y The Royal Society for the Prevention of Cruelty to Animals (RSPCA) ${ }^{19}$, por el riesgo de comprometer una buena valoración científica-ética de los procedimientos experimentales con animales, y la defensa de su cuidado y bienestar. En base a la descripción de la nueva Directiva, el grupo de bienestar animal se centraría básicamente en el estado del animal, manteniendo un rol de supervisión técnica y científica y asegurando que los principios de las 3 Rs son cumplimentados y promueven el bienestar y cuidado de los animales. Curiosamente en la nueva directiva no se cita la palabra "ética" como base de reflexión a la hora de valorar los proyectos con animales de experimentación. Contrariamente, en el actual sistema británico, el "Ethical Review Process "(ERP) tiene asignadas funciones bien definidas en estas áreas: 1) dar una valoración ética independiente a los solicitantes de proyectos de investigación, en particular en referencia al proceso de solicitud para la licencia animal de experimentación, reforzando y protegiendo el bienestar y cuidado de los animales; 2) dar apoyo al personal responsable del cuidado y mantenimiento de los animales, y a los investigadores trabajando en animales de experimentación, desde el área de bienestar animal y valores éticos ligados a dicho trabajo; 3) promover el uso de un análisis ético para dar consciencia de la importancia de defender el bienestar animal y promover iniciativas que favorezcan la aplicación de las 3Rs (Reducción del uso de animales, refinar los proyectos y buscar otras alternativas a la utilización de animales). Claramente la nueva Directiva no marca tanto énfasis en valoraciones éticas, centrándose más en valoraciones técnicos, aunque implícitamente siga manteniendo como objetivo principal la protección del bienestar animal. Esta ambigüedad en las valoraciones está siendo bastante criticada en el Reino Unido, ya que comprensiblemente las instituciones quieren seguir manteniendo su buena praxis y experiencias adquiridas a través del comité ético. El establecimiento de los Ethical Review Process (ERP) han jugado un papel muy importante en defensa del bienestar animal desde que su implementación en el Reino Unido en $1999^{\text {Xviii }}$, tal y como lo confirman varias instituciones británicas incluyendo RSPCA, LASA, Society of Biology, las universidades e instituciones gubernamentales. Si bien hay ciertas funciones del comité ERP que son transferidas al nuevo comité del bienestar animal, sus bases de

\footnotetext{
${ }^{19}$ Consultation on options for the transposition of European Directive 2010/63/EU on the protection of animals used for scientific purposes. RSPCA. August 2011.

http://content. www.rspca.org.uk/cmsprd/Satellite?blobcol=urldata\&blobheader=application\%2Fpdf\&blobkey=id \&blobnocache $=$ false $\&$ blobtable $=$ MungoBlobs \&blobwhere $=1233003379140 \&$ ssbinary $=$ true
} 
funcionamiento van mucho más allá de los aspectos técnico-logísticos de experimentación, tal y como describe la guía para la buena praxis del ERP publicada conjuntamente por LASA y el RSPCA ${ }^{20}$. Es importante clarificar que los conceptos de bienestar animal y ética no tienen la misma connotación. El concepto de bienestar animal es mucho más específico y se limita a los aspectos directamente relacionados con el bienestar físico/ general del animal. Por su parte, la valoración ética es un concepto mucho más amplio incluyendo aspectos éticos, legales, sociales, de responsabilidad civil y sobre la calidad del trabajo científico y valoraciones de coste/beneficio. En este contexto, el rol del comité ERP es mucho más amplio ya que potencia este amplio análisis ético como instrumento de valoración de los proyectos de investigación con animales de laboratorio para promover el bienestar de los animales. Asimismo se le atribuye un rol mucho más interactivo como foro de discusión de ámbitos relacionados con el uso de animales de laboratorios, estableciendo análisis prospectivos de coste y beneficio a la hora de valorar los proyectos. También se le atribuye un rol educativo y de promoción del dialogo con el público en general para educar y promover una actitud más crítica con respeto a la investigación con animales de laboratorio.

Otro aspecto que está creando bastante controversia es la falta de claridad a la hora de definir quién debe formar este grupo de bienestar animal. En la legislación vigente británica, el comité ético ERP debe incluir siempre a un veterinario responsable de los animales de experimentación y al personal responsable de su cría, cuidado y mantenimiento. Asimismo se valora muy positivamente que otros profesionales formen parte de dicho comité, por ejemplo, personal científico, profesionales de la ley y personal no relacionado con ciencia ni investigación animal. La consulta realizada por el gobierno británico ${ }^{\mathrm{s}}$ defiende claramente la participación del veterinario responsable de los animales de experimentación, y del personal técnico encargado de la cría y cuidado de los animales, ya que su formación y experiencia profesional son muy importantes a la hora de diseñar proyectos con animales de laboratorio y aconsejar sobre el bienestar animal y el diseño experimental. La participación de miembros del público en general, no directamente involucrados en la institución científica también está muy bien valorada porque refuerza la necesidad de mantener un dialogo interactivo con la sociedad y promover la educación en el área de investigación con animales. En este sentido la nueva directiva falla en concretar específicamente quien debe formar parte del grupo de bienestar y en base a que conocimientos se basa la selección de sus miembros. Asimismo, también se diluye el rol del ERP como un amplio foro de discusión y de educación en defensa del bienestar animal en las instituciones científicas. Hay que tener presente que los actuales comités éticos están muy bien considerados en los diferentes instituciones científicas, tal como lo refuerza el documento en su apoyo de la Federación Europea de Asociaciones Científicas de Animales de Laboratorio (FELASA) ${ }^{21}$, como un instrumento con un buen grado de autoridad para imponer y defender una buena praxis en defensa del bienestar animal.

\footnotetext{
${ }^{20}$ Guiding principles on good practice for Ethical Review Process. July 2010

http://www.lasa.co.uk/GP\% 20ERP\% 20J uly\% 202010\% 20print\% 20FI NAL.pdf

${ }^{21}$ Principles and practice in ethical review of animal experiments accros Europe 2005 
Finalmente, el comité ético ERP también cumple un importante rol dentro del sistema británico en supervisar y aconsejar la solicitud y modificaciones de las licencias personales y de proyectos de investigación con animales, antes de ser enviadas a la autoridad competente estatal. Este proceso es muy importante porque facilita la interacción con los investigadores y permite asegurar que los valores de defensa del bienestar animal y los principios de las 3Rs son considerados. También tiene un papel importante en referencia al seguimiento de los proyectos de investigación a través de la implementación de revisiones retrospectivas y de manera continuada de los proyectos ya aprobados, con el objetivo de constantemente mejorar la implementación de las 3Rs. Claramente el comité ético ERP juega tiene un papel muy importante dentro de las instituciones científicas, asegurando el cumplimiento de la legislación vigente en defensa del bienestar animal, y también en la formación y promoción de los valores éticos de defensa de los animales de laboratorio. A nivel logístico, el comité ERP juega un papel muy importante de mediador entre la autoridad estatal y el personal investigador trabajando en este campo incluyendo estudiantes, científicos, técnicos, administrativos etc..., estableciendo una dinámica de trabajo más práctica, flexible y eficiente con la administración. Esto se ve además facilitado por la participación del inspectorado de la autoridad estatal en el comité ERP, estableciendo así un buen foro de discusión entre investigadores y la administración.

\section{Conclusiones}

En los últimos años el sector médico-científico se ha involucrado mucho más en varios ámbitos sociales, políticos, mediáticos y de formación para asegurar la defensa del bienestar de los animales utilizados en experimentación, promoviendo la implementación de las 3Rs. Las normativas existentes han permitido proteger el uso responsable de animales de laboratorio, beneficiando los avances científicos. Asimismo ha permitido implementar una cultura de respeto y protección de los animales de experimentación, lo cual es particularmente importante entre las generaciones jóvenes de estudiantes e investigadores en el campo médico-científico. Sin duda esta política también ha mejorado mucho la percepción crítica de la sociedad en referencia al uso de animales en investigación biomédica. Es importante que durante este periodo de transposición las diferentes coaliciones de profesionales revisen la nueva directiva de un modo crítico y constructivo, y negociando conjuntamente con la autoridad estatal se aseguren que la nueva normativa mantiene los valores científicos y de defensa del bienestar animal. La interacción entre profesionales del área legal y administrativa con personal experto en investigación y en la utilización de animales y de defensa del bienestar animal es crucial para asegurar el buen impacto de la nueva normativa en bienestar animal y en ciencia. En este sentido es de alabar la gran participación de múltiples asociaciones del sector biomédico británico colaborando con el gobierno del país para asegurar una buena transposición de la normativa, en defensa de su ejemplar praxis en defensa del bienestar animal y la alta calidad de su investigación. 